Revue des patrimoines

16 | 2011

Le patrimoine militaire et la question urbaine

\title{
La caserne de la Visitation à Angers : réoccupation d'un ancien couvent de 1792 à 1904
}

Rose-Marie Le Rouzic

\section{OpenEdition}

Journals

Édition électronique

URL : http://journals.openedition.org/insitu/192

DOI : 10.4000/insitu.192

ISSN : 1630-7305

Éditeur

Ministère de la culture

Référence électronique

Rose-Marie Le Rouzic, «La caserne de la Visitation à Angers : réoccupation d'un ancien couvent de 1792 à 1904 », In Situ [En ligne], 16 | 2011, mis en ligne le 19 janvier 2012, consulté le 20 avril 2019. URL : http://journals.openedition.org/insitu/192 ; DOI : 10.4000/insitu.192

Ce document a été généré automatiquement le 20 avril 2019

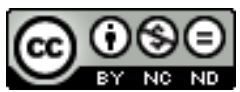

In Situ Revues des patrimoines est mis à disposition selon les termes de la licence Creative Commons Attribution - Pas d'Utilisation Commerciale - Pas de Modification 4.0 International. 


\title{
La caserne de la Visitation à
}

\section{Angers : réoccupation d'un ancien couvent de 1792 à 1904}

\author{
Rose-Marie Le Rouzic
}

1 La découverte en 2008 par l'Inrap ${ }^{1}$ d'une portion de l'ancien mur d'enclos du couvent de la Visitation à Angers, nous avait déjà conduits à nous intéresser aux bâtiments dans leur environnement, à leur devenir ${ }^{2}$ et à compléter les connaissances historiques sur l'ensemble du secteur. Notre approche concernera ici la période située entre 1792, date d'arrivée des militaires dans l'ancien couvent, et 1904, date de destruction de la caserne. Nous essayerons de suivre l'adaptation fonctionnelle des bâtiments en confrontant les textes à la documentation iconographique ${ }^{3}$.

\section{Le site}

\section{Le couvent de la Visitation}

2 L'ordre des Visitandines s'est installé à Angers au cours de la première moitié du XVII ${ }^{e}$ siècle ${ }^{4}$, dans un secteur extra-muros occupé dès l'époque médiévale par plusieurs propriétés ecclésiastiques. Les deux closeries de «Champs-Marais», acquises en 1643 pour douze mille livres, relevaient du fief du roi de France ${ }^{5}$ et dépendaient de la paroisse Saint-Germain en Saint-Laud ${ }^{6}$. La construction des bâtiments, débutée en 1644, s'est poursuivie jusqu'au XVIII e siècle à la faveur des rentes et donations perçues par les religieuses.

3 Un plan aquarellé, dressé en 1792 par l'architecte Jean-François de Marie et parfaitement conservé (fig. $\left.\mathbf{n}^{\circ} \mathbf{1}\right)$, nous donne une idée de la propriété. 
Figure 1

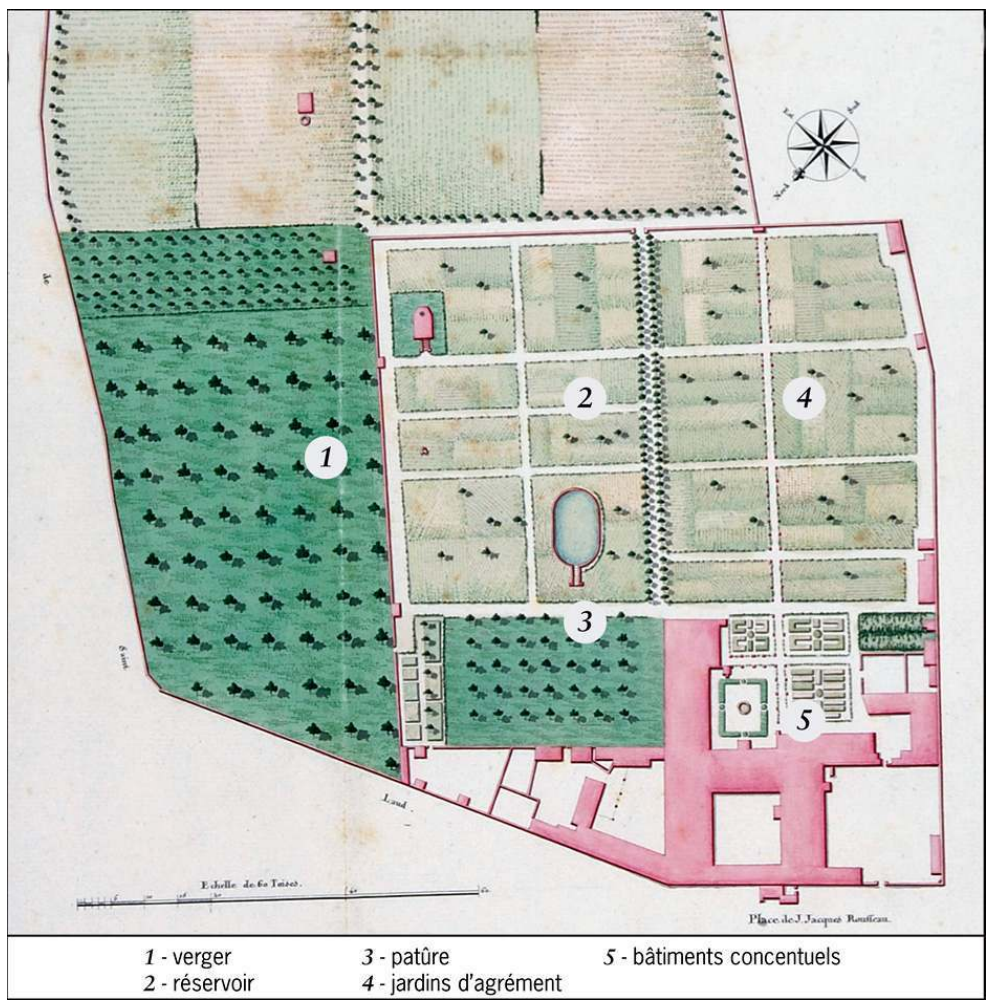

Détail du plan général du monastère de la Visitation d'Angers dans son état actuel par De Marie, le 5 mai 1792, I'an IV de la liberté, plan aquarellé couleur 63,5 x 49,8 cm. Arch. dép. Maine-et-Loire, 11 Fi 1077.

Phot. Éric Jabol, infographie Éléonore Rubington. ( Inrap.

Les bâtiments s'intègrent dans un grand parcellaire au découpage irrégulier correspondant aux terres des deux closeries acquises ${ }^{7}$. Ils s'alignent au nord-ouest sur la rue de Saint-Laud où l'entrée principale, bénéficiant d'un grand dégagement, forme une place ${ }^{8}$; celle-ci correspondait probablement, dès le Moyen Âge, à un carrefour entre le chemin de la Croix-Montaillé et celui de la Croix de Saint-Laud.

Le couvent, de plan carré, s'organise autour d'une cour centrale et est cantonné par trois autres cours dont une, formant un cloître (inachevé), donne sur des jardins. Autour de la cour des servitudes située à l'est, se trouvent les communs et une étable.

Le plan présente plusieurs espaces de jardins, un verger, une pâture et un grand clos, l'ensemble se déployant sur huit hectares.

\section{La caserne de la Visitation}

7 Préalablement à l'étude du site en tant que caserne, il est important de rappeler la situation complexe générée par la présence des garnisons dans la ville durant des décennies. En dépit de la décision prise, dès le règne de Louis XIV, d'élever des casernes dans tout le royaume, les habitants d'Angers supportent encore, à la veille de la Révolution, les lourdes charges imposées par les hébergements réguliers dus aux troupes. La réquisition des couvents, décidée en 1792 et dictée par les contraintes budgétaires de l'État, va brusquement résoudre ce problème du casernement. 


\section{Les projets}

\section{Réaménagement des bâtiments, création}

L'installation de bataillons dans des locaux initialement destinés à la vie claustrale, dont les besoins étaient tout autres que ceux d'une garnison militaire, impliquera très vite un remodelage de l'espace afin de l'adapter à l'usage d'une garnison de cavalerie.

Figure 2

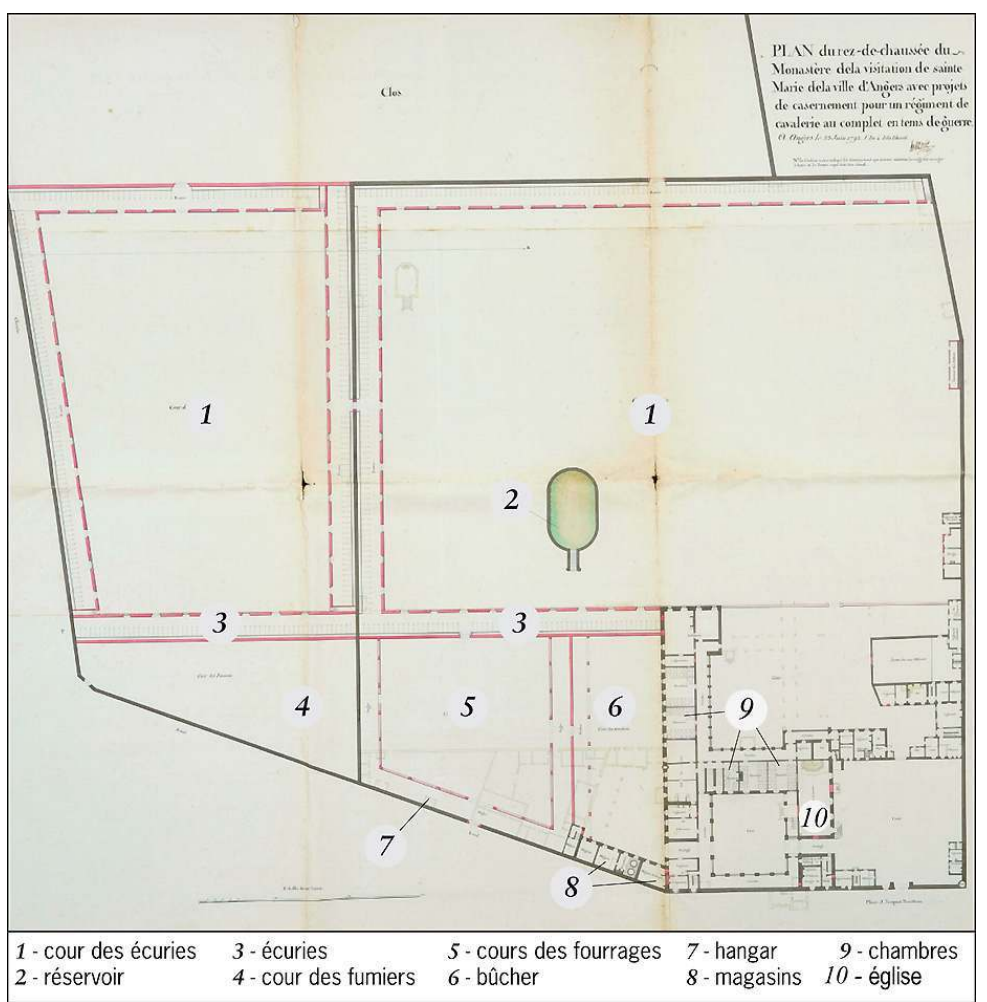

Plan du rez-de-chaussée du monastère de la Visitation de Sainte-Marie de la ville d'Angers avec projet de casernement pour un régiment de cavalerie au complet en temps de guerre, 23 juin 1792, I'an IV de la liberté. Arch. dép. Maine-et-Loire, 1 L $566^{12}$ bis.

Phot. Éric Jabol, infographie Éléonore Rubington. () Inrap.

Le site de la Visitation est immédiatement mentionné pour l'installation de cinq cents hommes et autant de chevaux, sans commune mesure avec sa réelle capacité d'accueil...9 Un plan dressé à cette période prévoit la future affectation des bâtiments, cours et communs « pour servir de casernement ». Le projet du rez-de-chaussée reproduit ici (fig. $\mathbf{n}^{\circ} 2$ ) fait état de quelques chambres dans la grande aile ouest ainsi que dans l'aile nord-est perpendiculaire à l'ancienne église, elle-même vouée à devenir « le magasin d'avoines ». Les plans des niveaux supérieurs ( $1^{\mathrm{er}}$ et $2^{\mathrm{e}}$ étages) ${ }^{10}$ révèlent des subdivisions internes correspondant à un souci d'optimiser au maximum la capacité de logement. Mais même les chambrées de six ou huit lits ne peuvent répondre à l'effectif annoncé de cinq cents hommes... Tout au plus, la caserne en recevait-elle la moitié. En ce qui concerne les écuries et l'entretien des chevaux, le projet comporte la création de plusieurs séries de boxes encadrant les anciennes zones de cours, jardins ou verger de la propriété, transformées pour la circonstance en aires de stockage (fourrage ou fumier). L'intérêt 
présenté par ce site résidait justement dans son envergure. Il n'est cependant ici question que du premier parcellaire, le grand clos que l'on devine en haut des deux documents étant probablement identifié pour servir aux manœuvres.

Un autre projet, datant également de la fin du XVIII ${ }^{\mathrm{e}}$ siècle, était encore plus ambitieux puisqu'il prévoyait de fondre les deux sites de la Visitation et de Sainte-Catherine ${ }^{11}$ et d'inclure d'autres propriétés mitoyennes. Ce programme prestigieux à l'ordonnancement très symétrique ne pouvait aboutir du fait de la conjoncture économique de l'époque mais témoigne de la volonté de créer un vrai casernement (fig. $\mathbf{n}^{\circ} \mathbf{3}$ ).

\section{Figure 3}

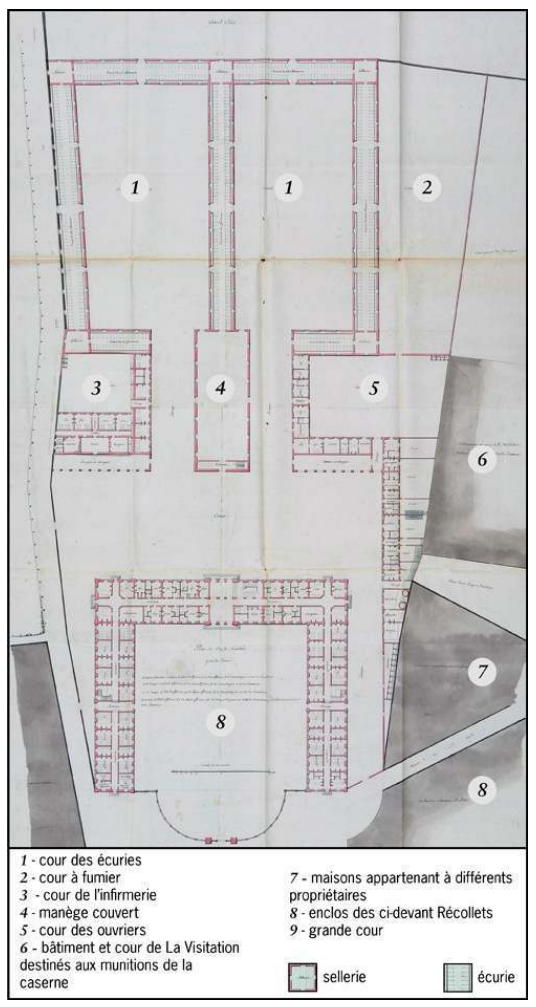

Projet d'un corps de caserne à construire à Angers sur l'emplacement des ci-devant monastères de Sainte-Catherine et de la Visitation pour le logement de cavalerie de 4 escadrons au complet en temps de guerre conformément à la loi du 21 février 1793 sur l'organisation de l'armée. Arch. dép. Maine-etLoire, 1 L $566^{12}$ bis.

Phot. Éric Jabol, infographie Éléonore Rubington. () Inrap.

11 En vertu du décret de la Convention nationale, une levée massive d'hommes de cavalerie s'annonce dans le pays en 1793 , dont une centaine est assignée au district d'Angers ${ }^{12}$. Cette décision va contraindre l'hôpital des Incurables, déjà installé dans les locaux de la Visitation, à cohabiter durant des années avec les militaires ${ }^{13}$.

12 L'absence de fonctionnalité des monastères reconvertis en casernes provoque d'ailleurs de nombreux atermoiements à Angers. Des projets de rénovations ou de réaménagements vont ainsi se porter successivement sur les bâtiments des abbayes de Saint-Serge, Toussaint et Saint-Nicolas, et sur les couvents de Sainte-Catherine et des Minimes. Les dégradations parfois commises par les bataillons conduiront l'administration du Directoire à suggérer des mesures de surveillance ${ }^{14}$. À une époque où de nouveaux décrets remettaient les casernes à la charge des communes ${ }^{15}$, le dessein d'un établissement de 
cavalerie dans l'ancienne abbaye du Ronceray, peu adaptée, va échouer au profit de la Visitation où l'implantation d'un régiment s'est, en réalité, vraiment effectuée dès $1810^{16}$.

Les avantages de ce site «isolé et très aéré, bien desservi par de larges chemins et spacieux pour les manœuvres » ne manquent pas d'être exposés au préfet en 1811. Un extrait du cadastre de 1809 recalé sur le plan actuel permet de situer le parcellaire et d'appréhender son étendue (fig. $\mathbf{n}^{\circ} \mathbf{4}$ ).

Figure 4

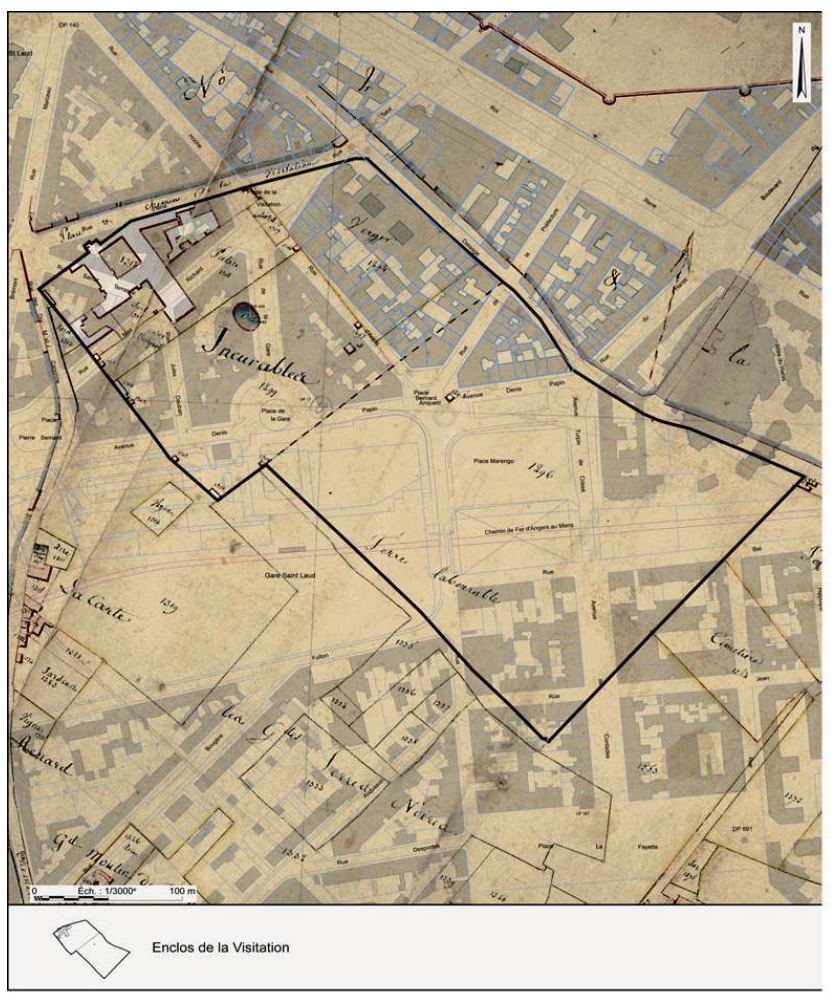

Extraits du cadastre d'Angers de 1809, feuille C3 sud. Arch. dép. Maine-et-Loire, 3 P 4/7/22 et du cadastre d'Angers de 2003.

Infographie Didier Pfost. (c) Inrap.

En 1812, le site de la Visitation est décrit comme « un local immense, nécessitant quelques travaux et nouvelles distributions ». On prévoit d'y édifier un petit pavillon et des bâtiments accessoires (boutiques, cellier) mais surtout des écuries, propres à héberger 750 chevaux et que l'on pourrait adosser à « un mur de clôture jugé en excellent état $~{ }^{17}$.

À cette époque, le Ministère de l'Intérieur suggère à la Ville d'acquérir les bâtiments et jardins qui sont alors propriété des Hospices, afin d'opérer les transformations nécessaires à la caserne. Les besoins s'inscrivent dans un contexte d'urgence nationale, en pleine guerre napoléonienne ; il est d'ailleurs demandé de réquisitionner tous les chevaux des particuliers afin d'équiper chaque soldat dans les dépôts ${ }^{18}$. Le maire fait encore valoir en 1814 le potentiel du «local de la Visitation » jugé supérieur à celui du Ronceray (espace pour 750 chevaux, abreuvoir facile d'accès, vastes issues) ${ }^{19}$. La commission administrative des hospices d'Angers consent à céder les bâtiments et l'enclos à la Ville pour le prix de 48000 francs qu'elle remboursera annuellement ${ }^{20}$. Celle-ci s'engage également, en échange de l'obtention du Ronceray où elle souhaite voir établir l'école des Arts et Métiers, à compléter le casernement de la Visitation pour recevoir deux bataillons. 
Le transfert de l'Infanterie de Saumur est ainsi autorisée ${ }^{21}$ mais l'arrivée de plusieurs centaines d'hommes implique des réfections dans l'ancienne église car l'armée souhaite utiliser l'espace pour ses classes de théorie et disposer d'un local pour des ouvriers.

La lenteur de l'installation ne manque pas d'irriter vivement la commission administrative des Hospices. Un tableau réalisé par la $22^{e}$ division militaire pour le département, réactualise par ailleurs le projet d'écuries maçonnées, adossées à un mur de clôture ${ }^{22}$. Nous sommes loin de l'idée première d'écuries cantonnant les cours principales et la difficulté de mener le projet à terme transparaît à travers tous les échanges de courriers entre les différents partis.

En ce qui concerne l'aménagement général des bâtiments de la caserne, un procès-verbal établi en 1818 révèle peu de changement ${ }^{23}$. Le rez-de-chaussée ne présente que deux chambres avec cheminées donnant sur la première cour et cinq sur l'ancien cloître. Si la description du premier étage fait état d'une dizaine de chambres, les greniers, au deuxième étage, n'en possèdent pas, même si l'un d'entre eux est signalé pour avoir servi jadis de logement, probablement lors du séjour des Incurables. Ces indications témoignent de l'inexistence du casernement au regard du projet initial.

En réalité, faute de moyens financiers, la situation demeure lacunaire en 1821 et l'on évoque alors la possibilité d'un transfert des troupes d'infanterie vers l'ancien couvent de Saint-Nicolas. Le site de la caserne de la Visitation est cependant conservé et nombre de réparations indispensables y sont signalées en 1838 (étayages de voûtes de caves, remplacement de chaînages d'angles) ${ }^{24}$. L'administration des hospices cesse alors de jouir définitivement de l'enclos où elle avait conservé quelques parties. Cet enclos va faire l'objet d'un bail à ferme, comportant non seulement les bâtiments mais aussi tous les jardins. Entre 1839 et 1840, s'y annonce en effet l'adjudication aux enchères publiques pour trois ans de plusieurs lots ${ }^{25}$ dont un comprenant le grand «jardin des dames». Le produit des terrains revient encore, à ce moment-là, à l'administration municipale. La Ville précise qu'elle ne souhaite pas réaliser de travaux dans les bâtiments pendant la durée du bail. Les procès-verbaux d'adjudication ne s'attardent pas à détailler le bâti mais on y trouve parfois quelques indications telle la mention d'une "porte charretière et d'une petite porte dans la rue de la Visitation $»^{26}$. La lithographie pittoresque réalisée en 1844 par Louis-Jules Arnout (fig. $\mathbf{n}^{\circ}$ 5), document aquarellé représentant une vue d'Angers en ballon, ne nous livre pas l'ensemble de "l'enclos de la Visitation ", tel qu'on le voit parfois encore nommé mais une illustration de la partie nord. Si nous pouvons douter de la précision de sa représentation pour les bâtiments (la galerie du cloître n'est pas figurée, il manque un retour d'aile dans les bâtiments principaux), nous trouvons ici des indications intéressantes quant aux anciennes zones agricoles apparemment préservées, notamment la partie jardin comportant le grand réservoir mais aussi l'ancienne pâture et les communs. En revanche, nous notons la disparition du grand verger au profit, semble$\mathrm{t}$-il, d'une aire de stockage ou atelier, dotée de petites constructions en bordure du chemin de terre ${ }^{27}$. À cette période, la physionomie générale de l'ancien couvent n'a donc vraisemblablement pas beaucoup évolué. 
Figure 5

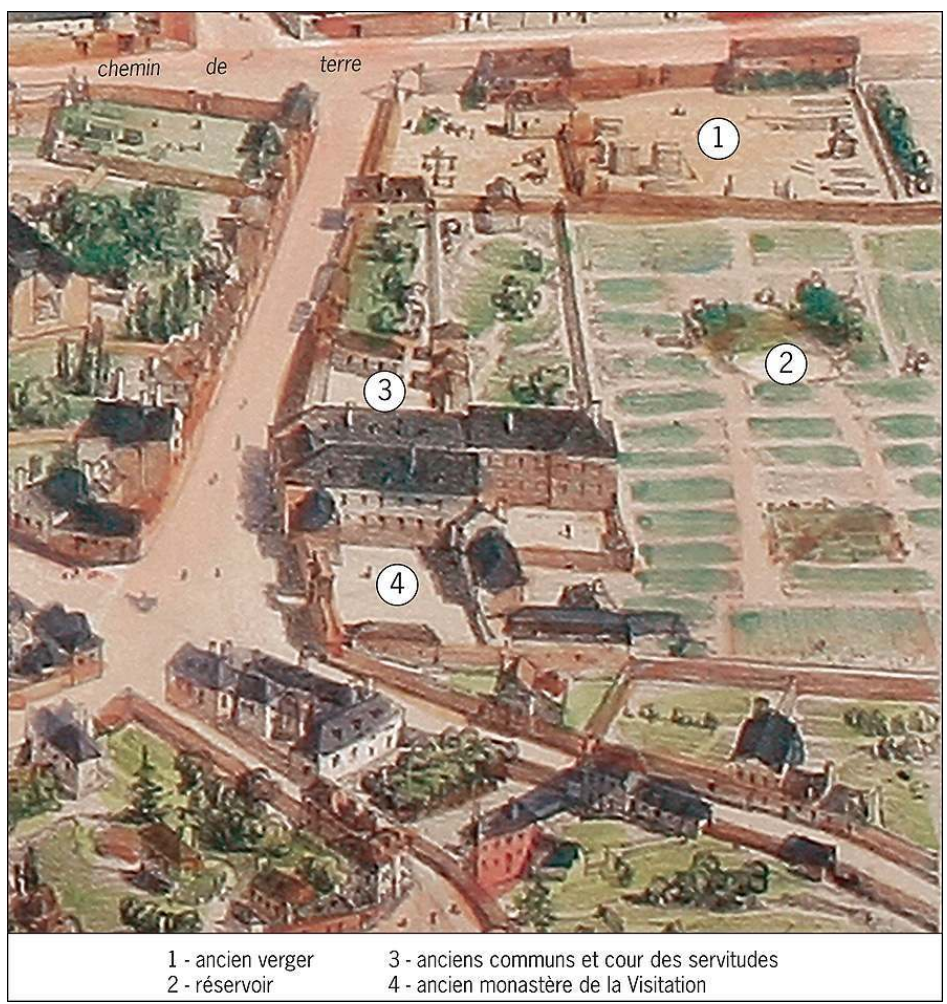

Détail de la vue d'Angers en ballon par J. Arnout, 1844. Arch. mun. Angers, O 2 Fi 335.

Phot. Bruno Amiot, infographie Didier Pfost et Éléonore Rubington. (c) Inrap.

\section{Le dépôt de remonte}

19 À cette époque, Angers possède deux casernes de cavalerie : la Visitation et l'Académie ${ }^{28}$, totalisant 1142 hommes et 141 chevaux. Elle est la deuxième ville la plus importante sur le plan militaire après Nevers mais dispose de moins de chevaux qu'ailleurs du fait de l'inadaptation de ses bâtiments ${ }^{29}$. Le préfet est sollicité à maintes reprises pour le projet touchant aux écuries de la caserne, le besoin d'une succursale de remonte devenant urgent après la mortalité importante des chevaux sur le site de l'Académie ${ }^{30}$. L'incendie survenu sur place en 1844 est l'occasion de dénoncer l'insuffisance chronique du site et un devis pour de nouvelles constructions est fourni l'année suivante. Le projet d'un établissement de remonte, préoccupation apparue dès la fin du XVIII e siècle à Angers, a donné lieu à diverses propositions entre 1839 et 1843 comme l'atteste la quinzaine de projets conservés ${ }^{31}$. Certains expriment un réel souci de volumétrie, conjuguant critères fonctionnels et esthétiques (fig. $\left.\mathbf{n}^{\circ} \mathbf{6}\right)$ (fig. $\left.\mathbf{n}^{\circ} \mathbf{7}\right)$. 


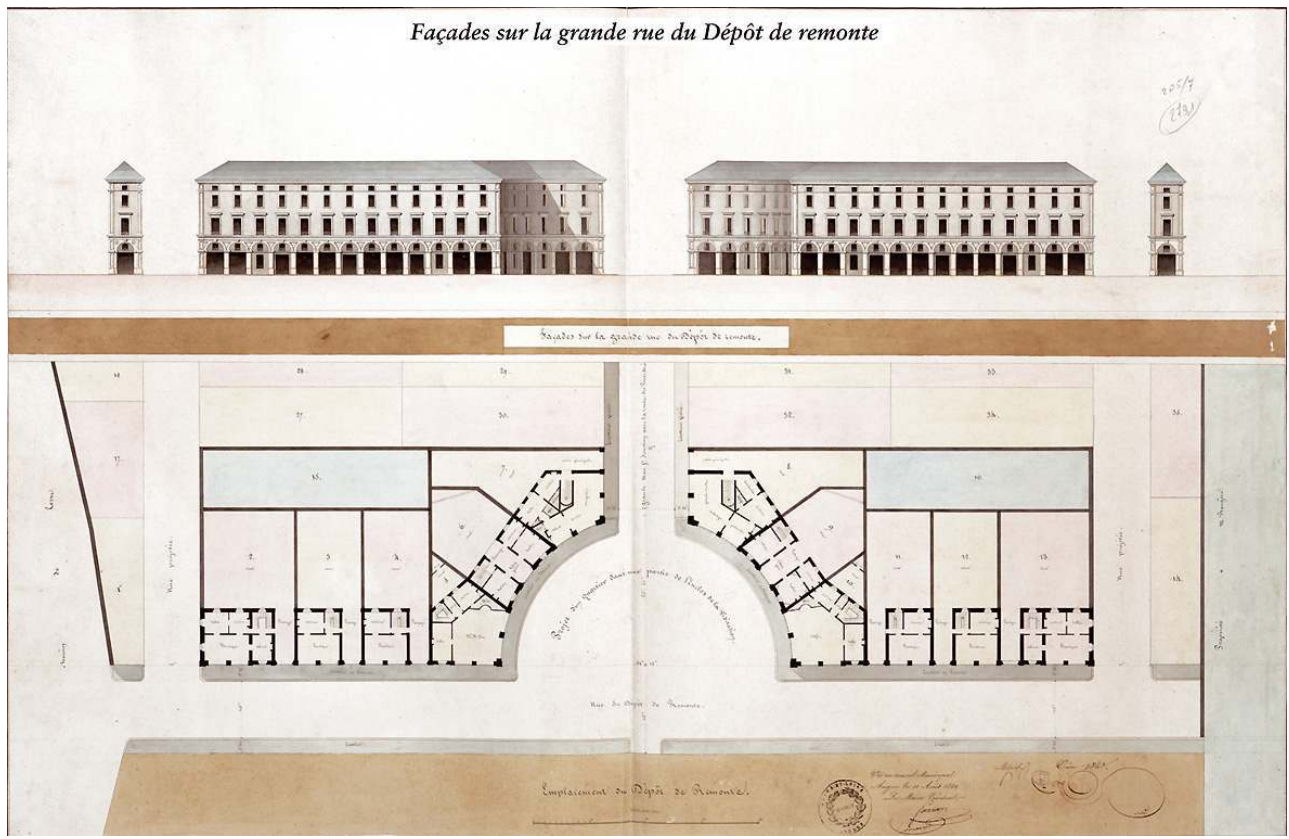

Angers, enclos de la Visitation, caserne et dépôt de remonte 1839-1841. Élévation sur la grande rue, plan du dépôt de remonte, juin 1840, Villers architecte, papier, plume et aquarelle, 94,4 x 62,7 cm. Arch. dép. Maine-et-Loire, 90 J 2179.

Phot. Éric Jabol, infographie Éléonore Rubington. (C) Inrap.

En 1843 l'autorisation d'un dépôt de remonte est officiellement donnée et le financement théoriquement assuré par l'aliénation d'une portion des terrains de la Visitation; mais l'implantation du chemin de fer dans l'ancien enclos obligera à renoncer à ce site. 


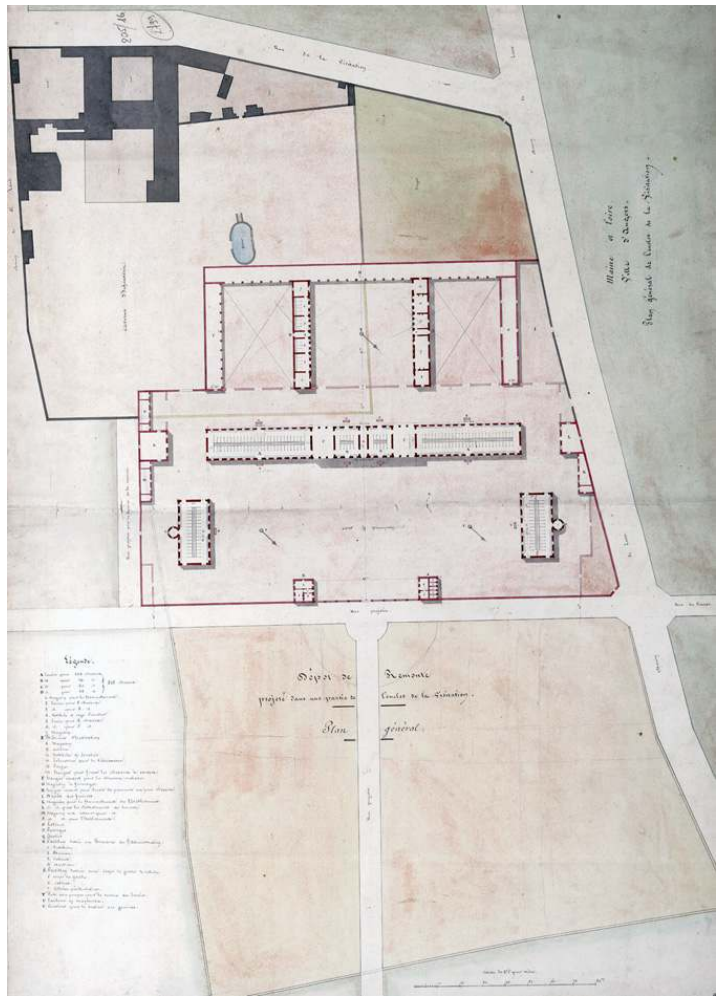

Angers, enclos de la Visitation, caserne et dépôt de remonte 1839-1841. Plan général de l'enclos, sd, non signé, papier, plume et couleur, 94 × 66,10 cm. Arch. dép. Maine-et-Loire, $90 \mathrm{~J} 2175$.

Phot. Éric Jabol, infographie Éléonore Rubington. (c) Inrap.

\section{L'agrandissement de la caserne}

À la fin de l'année 1849, la direction du Génie à Nantes réclame à la Ville d'Angers « de remettre au Ministère de la guerre les terrains compris entre la caserne de la Visitation, la rue de l'Embarcadère et le chemin de ceinture de la gare ", et affirme "qu'elle fera enclore sans délai les dits-terrains de murs de 3,30 m de haut à l'extérieur et qu'elle garantira la construction d'une caserne... remise au Génie dans un délai de trois ans " $^{32}$. Un projet décrit alors le futur ouvrage : un mur en moellons de $35 \mathrm{~m}$ x 2,66 m pour $0,50 \mathrm{~m}$ d'épaisseur, et incluant des remplois ${ }^{33}$.

Le site de la Visitation prend son allure définitive à partir de 1851, date de démarrage d'un grand chantier pour la construction d'un corps de caserne destiné à 720 hommes. Le projet $^{34}$ comprend la démolition de petits bâtiments se trouvant dans les nouveaux terrains annexés à la caserne et l'amélioration de la façade principale du vieux bâtiment donnant sur la rue des Lices ${ }^{35}$. Il est spécifié que la maçonnerie "devra être dérasée à hauteur du sol et qu'il faudra abattre un vieux portail délabré, à l'entrée du carrefour »; l'ensemble des matériaux étant destiné à la vente en un seul $\operatorname{lot}^{36}$.

L'agrandissement de la caserne va consister en la réalisation d'un bâtiment de plan en L dont toutes les dépenses de maçonnerie seront achevées en 1852, et celles des planchers, plafonds et dallages en 1854 ; l'occupation des nouveaux bâtiments se fera dès l'année suivante ${ }^{37}$. 

utilisés tant pour le bâti et les cours (grès, tuffeau blanc et gris, briques doubles, ou " gros-billots ", dalles de granit, carreaux de terre cuite) que pour la charpente (chêne, sapin du Nord) ou les planchers (chêne du Nord, sapin rouge). Il intègre également les modifications à faire sur les parties anciennes (démolition de vieilles maçonneries pour percement de baies) mais sans les situer. Ce document n'élude pas les plantations et fournit des informations sur les essences prévues (ormeaux, tilleuls, marronniers, platanes). prennent forme en 1855 pour «le casernement deux escadrons de lanciers » et une quatrième, en 1856. Ces baraquements provisoires (deux doubles pour 200 chevaux, un pour 26 chevaux) se situent sur un terrain de $600 \mathrm{~m}^{2}$ compris entre le chemin de terre et la rue d'Anjou, et nécessitent de grosses réparations dès $1861^{39}$.

En définitive, l'échec du projet d'une grande caserne de cavalerie à la Visitation où ne demeurera que le $135^{\mathrm{e}}$ régiment d'infanterie, va trouver une compensation dans l'édification en 1862, d'un vrai quartier neuf au sud de l'enclos : la caserne du Clon ${ }^{40}$.

Figure 8

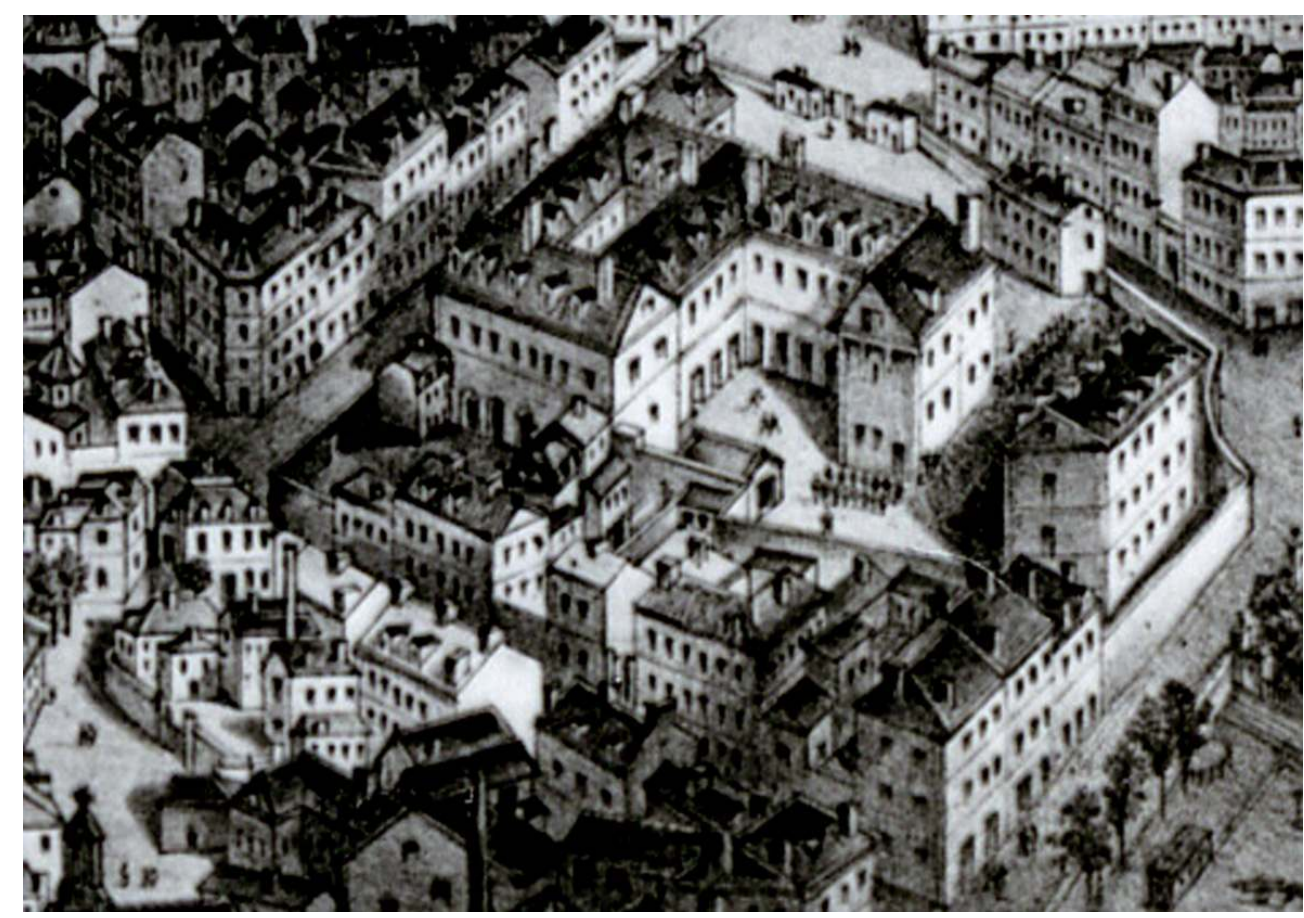

Détail de la vue d'Angers en 1878, vue prise en ballon. Paris, imp. Lemercier et Cie, Arch. mun. Angers, $1 \mathrm{Fi} 1578$.

Phot. Bruno Amiot, infographie Didier Pfost. (c) Inrap.

27 La vue en ballon d'Angers réalisée en 1878 (fig. $\mathbf{n}^{\circ} \mathbf{8}$ ) illustre assez bien - toutes proportions gardées - l'aspect général de la caserne de la Visitation, ensemble rogné au fil des décennies par une urbanisation croissante liée à la création du chemin de fer tout proche. La grande cour d'entrée, flanquée de deux pavillons et aménagée au nord-est sur la nouvelle place de la Visitation, a investi l'ancienne pâture et la cour des servitudes. Les

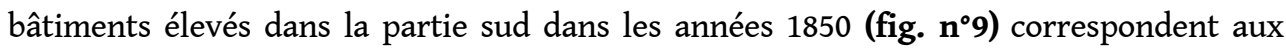
grands programmes architecturaux militaires lancés partout en France dans les 
《 casernes-monastères $»^{41 . ~ \AA ̀ ~ A n g e r s, ~ l ' e s p a c e ~ i n s u f f i s a n t ~ p o u r ~ l e s ~ m a n œ u v r e s ~ e t ~ l ' e n t r e t i e n ~}$ coûteux du bâti ancien, majoritaire, vont conduire inexorablement à la disparition de l'ensemble après la création de plusieurs établissements militaires à la périphérie de la ville ${ }^{42}$.

Figure 9

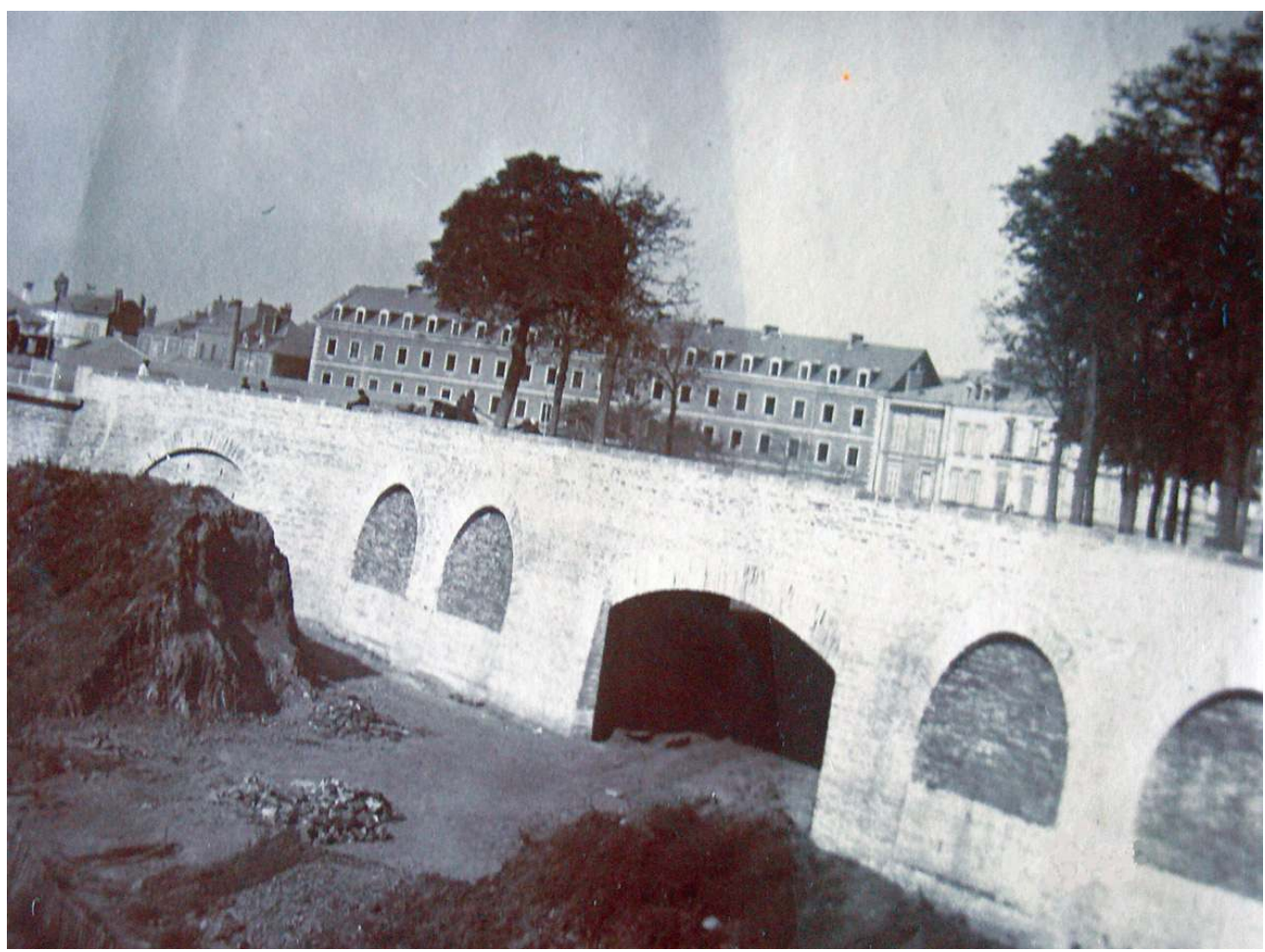

Angers, vue de la caserne de la Visitation prise de la rue Bel-Air. Achèvement du tunnel de la place Marengo pour le raccordement du petit Anjou, 1896. Arch. dép. Maine-et-Loire, 4 Fi 1758/1.

Phot. Rose-Marie Le Rouzic/Inrap, infographie Éléonore Rubington. () Inrap.

La caserne est abandonnée en 1903. Le maire désirait permettre le lotissement total ou partiel des terrains promis à la vente et la démolition débute au début de l'année 1904 ; mais auparavant, le Ministère de la guerre évoque un projet de transfert de la Manufacture militaire, alors située dans la tour Saint-Aubin, sur le site, depuis rebaptisé " caserne Desjardins », en hommage à un général d'Empire. L'estimation coûteuse de ce projet ne séduit pas la Ville et la destruction se poursuit, libérant de grands espaces pour la création d'une nouvelle rue (fig. $\mathbf{n}^{\circ} \mathbf{1 0}$ ) et de nouveaux îlots. Le quartier reste organisé autour de la place de la Visitation, désormais ancrée dans le paysage d'Angers. 
Figure 10

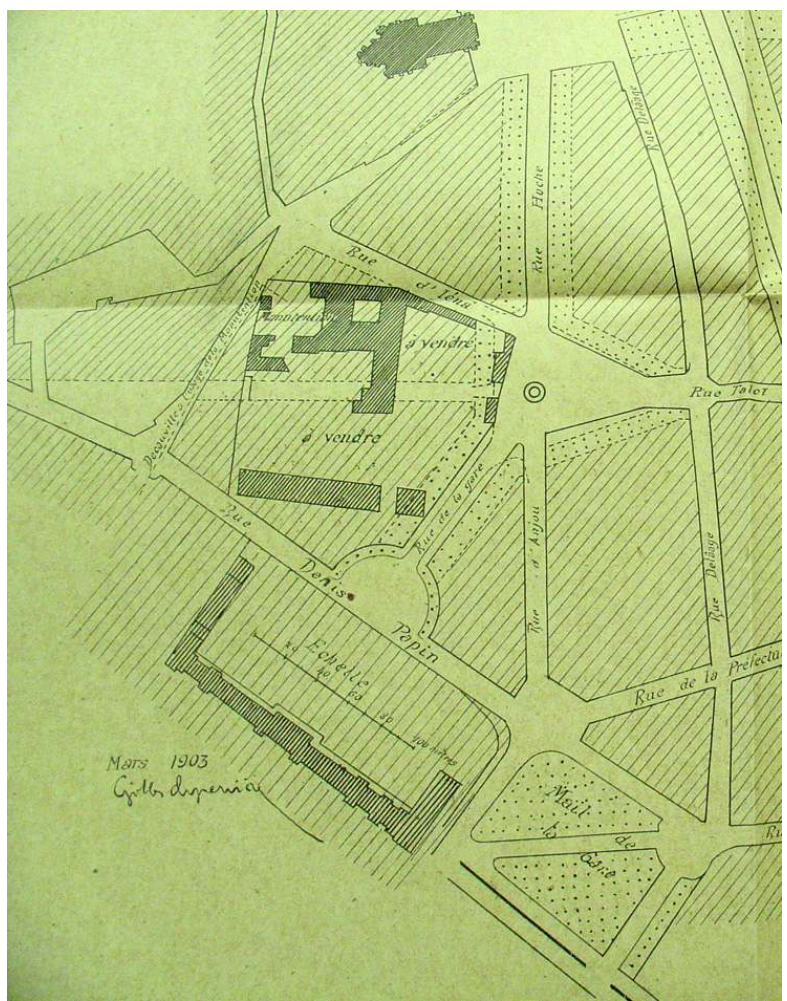

Détail d'un plan du secteur de la Visitation, mars 1903. Arch. mun. Angers, 10878.

Phot. et infographie Rose-Marie Le Rouzic. (C) Inrap.

\section{NOTES}

1. - GUERIN, Frédéric. Angers-rue de Létanduère, rapport de fouilles, INRAP/SRA des Pays-de-LaLoire, Nantes, 2008.

2. - LE ROUZIC, Rose-Marie. L'enclos de la Visitation d'Angers (du XVII ${ }^{\mathrm{e}}$ au début du XX ${ }^{\mathrm{e}}$ siècle), Archives d'Anjou. Mélanges d'histoire et d'archéologie angevines, $\mathrm{n}^{\circ} 13$, décembre 2009, $\mathrm{p}$. 121-135.

3. - Nous remercions ici Mme É. Verry, directrice des Arch. dép. de Maine-et-Loire, M.-S. Bertoldi, conservateur en chef des Arch. mun. d'Angers, M. Denis, service du Patrimoine de la ville d'Angers.

4. - Lettres patentes du roi Louis XIII en 1636.

5. - Un document de 1397 figurant dans les titres de propriétés de la Visitation mentionne que ledit «Champs-Marais » était réputé du domaine du roi, hors du domaine du roi de Sicile et duc d'Anjou (Bibl. mun. Angers, ms 888).

6. - Cette paroisse s'étendait extra-muros du château d'Angers jusqu'aux limites de la paroisse de Saint-Gemmes-sur-Loire. 
7. - Il est possible d'ailleurs d'envisager une imbrication de propriétés à une époque ancienne en relation avec les domaines mitoyens de Terre noire et de La Carte; une étude des fonds d'archives pour la période médiévale préciserait le paysage foncier d'alors.

8. - Dite d'ailleurs " place de la Visitation" au tout début du XIX ${ }^{\mathrm{e}}$ siècle après s'être nommée «place Jean-Jacques Rousseau » après la Révolution.

9. - A. D. Maine-et-Loire, $1 \mathrm{~L} 566{ }^{12}$ bis.

10. - Voir aux A. D. Maine-et-Loire, classés sous la même cote $1 \mathrm{~L} 566^{12}$ bis.

11. - Couvent des Bernardines, fondé au XVII ${ }^{\mathrm{e}}$ siècle de l'autre côté de la rue Saint-Laud.

12. - A. D. Maine-et-Loire, 2 L 91, procès-verbaux de casernement pour l'infanterie et la cavalerie dans divers monastères.

13. - L'hôpital des Incurables a été fondé en 1734 par Mme veuve Decazeaux, retirée à l'abbaye du Ronceray. Son projet initial devait s'inscrire dans l'enceinte de l'hôpital général d'Angers, mais la mauvaise gestion de l'établissement provoqua le transfert au «Clos du Présidial », situé paroisse Saint-Martin. Après un incendie causé par les troupes vendéennes, un nouveau déplacement fut envisagé dans l'urgence en 1793, cette fois vers la Visitation.

14. - Arch. mun. Angers, $2 \mathrm{H} 54$.

15. - POTIN, Philippe. Angers et la présence de l'armée, 1873-1914. Archives d'Anjou. Mélanges d'histoire et d'archéologie angevines, $\mathrm{n}^{\circ}$ 12, 2008, p. 177-195.

16. - PORCHET, Alain. L'armée dans la ville à Angers et en Anjou au XIX ${ }^{\mathrm{e}}$ siècle, Mémoire de DEA d'histoire contemporaine, Université d'Angers, 1996, p. 75.

17. - A. D. Maine-et-Loire, 207 art. 40, registre des délibérations du conseil municipal, séance du 12 janvier 1812.

18. - A. D. Maine-et-Loire, 2 R 4.

19. - Archives du Génie à Angers.

20. - A. M. Angers, 2 H 49.

21. - A. D. Maine-et-Loire, 207 40, lettre du Ministère de la guerre, 8 mai 1815.

22. - A. D. Maine-et-Loire, 207 40, registre des délibérations. On ne bâtira en réalité que des écuries en bois.

23. - A. M. Angers, $2 \mathrm{H}$ 43, procès-verbal.

24. - A. M. Angers, 2 H 41, bâtiments militaires dégâts occasionnés, arrêts, états des lieux, rapports.

25. - A. M. Angers, $4 \mathrm{~N}$ 6, biens communaux, terrains de la Visitation (1831-1864).

26. - A. D. Maine-et-Loire, 20741.

27. - Actuelle rue Delaâge.

28. - Ancienne Académie royale d'équitation.

29. - DESOEUVRE, Malika. Les casernes militaires à Angers de 1789 à 1794, Mémoire de maîtrise d'histoire, Université d'Angers, 2002, p. 73.

30. - A. D. Maine-et-Loire, 20740.

31. - Fonds des A. D. Maine-et-Loire, plans d'architectes en ligne.

32. - Archives du Génie à Angers.

33. - A. D. Maine-et-Loire, 20741.

34. - A. M. Angers, 2 H 49.

35. - Actuelle rue Talot.

36. - A. D. Maine-et-Loire, 20741.

37. - A. M. Angers, $2 \mathrm{H} 49$.

38. - A. D. Maine-et-Loire, $2 \mathrm{R} 21$.

39. - A. M. Angers, 2 H 49, voir « comptes sommaires des dépenses » émanant de la direction du Génie à Nantes.

40. - Occupait l'actuelle place Lafayette et fut baptisée successivement caserne Desjardins et caserne d'Espagne. 
41. - Voir dans la base Mérimée : notice IA77000437.

42. - Caserne du Clon, caserne Langlois, caserne Éblé dont les noms changent au XX ${ }^{\mathrm{e}}$ siècle.

\section{RÉSUMÉS}

La caserne de la Visitation à Angers fait partie des nombreuses « casernes-monastères » installées après la Révolution de 1789 dans les bâtiments religieux évacués. Elle se caractérise par la pérennité de son occupation, favorisée par un emplacement de choix et un vaste parcellaire. Les nombreux projets architecturaux et tractations ainsi que les différents corps d'armée qui s'y sont succédé durant une soixantaine d'années témoignent de l'importance de ce site que le projet de gare va partiellement amputer dans les années 1850. Avec l'avènement de nouveaux quartiers militaires en périphérie urbaine, à la fin $\mathrm{du}^{\mathrm{XIX}}{ }^{\mathrm{e}}$ siècle, la caserne connaît progressivement l'abandon puis la destruction en 1904.

The garrison house of La Visitation in Angers is one of many 'monastery-barracks' installed in religious buildings evacuated after the 1789 Revolution. It is characterized by its continuous occupation, taking advantage of a special location and of a large parcel of land. The successive architectural projects and negotiations as well as the presence of various army corps over sixty years, show the importance of the site which was partially amputated by the railway station project in the 1850s. With the construction of new military buildings on the outskirst of the town, in the late nineteenth century, the barracks were gradually abandoned and finally destroyed in 1904.

INDEX

Mots-clés : caserne, couvent, Visitation, Angers

Keywords : barracks, convent, Visitation, Angers

\section{AUTEUR}

\section{ROSE-MARIE LE ROUZIC}

assistante d'études à l'Institut national de recherches archéologiques préventives, région Paysde-la-Loire Rose-marie.le-rouzic@inrap.fr 